\title{
Estimation of Vertical Jump Height Using Nintendo Wii Remote IR Camera
}

\author{
Bhanupol Klongratog ${ }^{1}$,Warit Pengto ${ }^{1}$, Todsaporn Wornkert $^{1}$, and Anupong Srongprapa ${ }^{1}$ \\ ${ }^{1}$ Department of Physics, Faculty of Science, King Mongkut's Institute of Technology Ladkrabang, Bangkok, Thailand
}

\begin{abstract}
In this article, we proposed to measure the heights of countermovement jumps which are recorded in term of vertical leap by using the Wii Remote infrared camera. According to the physical principles, positions of the movement were detected based on the rules regarding conservation of energy, motion under gravity, and coordinate system. The obtained results were compared with that of the slowmotion measurements. The experiment involved 30 basketball players whose jump results were slightly deviated from the vertical measurement of the coordinate system. Therefore, the results should be calibrated each time the new system is installed.
\end{abstract}

\section{Introduction}

Sports science has been an integral part of many sports including the aspects of nutritional requirement, sportswear, and playing techniques that make the most capable athlete. Sport science innovations helps to reduce resistance between skin and the water for swimsuits [1], improving technique that helps to achieve higher jumps for basketball [2], volleyball and soccer players. The sport science knowledge is also extended to the field of humanoid development [3]. There are different methods that are used to measure the height of jumping including time taken to calculate the height of a vertical jump by using ground test strips to detect jumping and landing marks. Strain gauge, electrical switch [4-9], accelerometer [10-11] and optoelectronic [12] are also applied to above measurements. Training patterns may benefit from the measurements of jumping because athletes can be trained to jump more effectively. To achieve this, collected statistical data of each athlete will be analysed. Height measurements of jumping is related to a countermovement Jump using Wii Remote which is used in many research projects including virtual reality, tracking systems, control systems, and clinical tests [13-16]. The participants' waist-height is the level of movement which is used in measuring this vertical jump. To conduct experiments, a belt fitted with an infrared lamp at the buckle is attached to the waist position of participants. Then, an infrared camera located inside the Wii remote detected the position of participants once the jump is performed. The detected position was subsequently analysed by relevant physical equations such as the rules of energy conservation vertical movement and coordinate system. All of the analysis were processed by using computer program.

\subsection{System design}

It is important to eliminate interfering noises during the image processing. Furthermore, any signal or light that may interfere with the measurement system must also be eliminated. Installation stage using the infrared camera in the Wii remote mask should be at the position of 23 degrees of vertical angle [17] as shown in Figure 1. As per the height of the Wii remote above the ground (a.) 1 meter distanced from the Wii remote system to the participants must be maintained (b.) 2 meters with measuring device based on resolution of 1 centimeter.

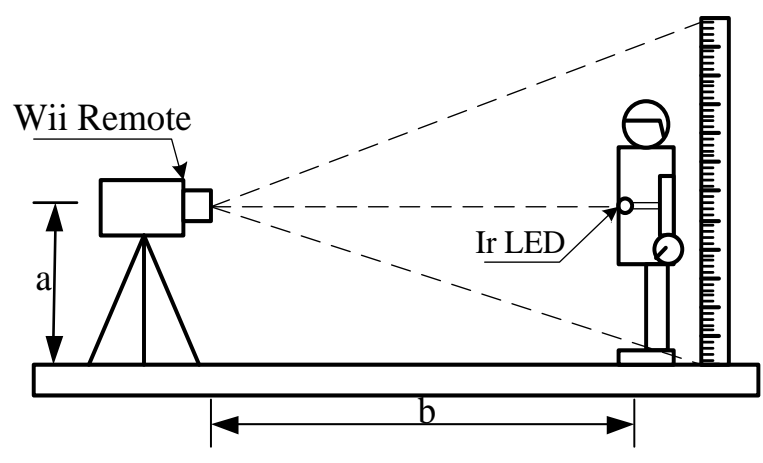

Fig. 1. Experimental setup.

\subsection{System testing}

Static testing system, according to the testing system in Figure 1, tests were conducted to evaluate effectiveness of image processing system using infrared cameras. infrared LED were installed at 25 different coordinate points on the backgrounds behind participants as per Figure 2. This experiment required 15 repeated tests.

\section{Materials and methods}




\begin{tabular}{|l|l|l|l|l|}
\hline $\mathrm{X} 1, \mathrm{Y} 5$ & $\mathrm{X} 2, \mathrm{Y} 5$ & $\mathrm{X} 3, \mathrm{Y} 5$ & $\mathrm{X} 4, \mathrm{Y} 5$ & $\mathrm{X} 5, \mathrm{Y} 5$ \\
\hline $\mathrm{X} 1, \mathrm{Y} 4$ & $\mathrm{X} 2, \mathrm{Y} 4$ & $\mathrm{X} 3, \mathrm{Y} 4$ & $\mathrm{X} 4, \mathrm{Y} 4$ & $\mathrm{X} 5, \mathrm{Y} 4$ \\
\hline $\mathrm{X} 1, \mathrm{Y} 3$ & $\mathrm{X} 2, \mathrm{Y} 3$ & $\mathrm{X} 3, \mathrm{Y} 3$ & $\mathrm{X} 4, \mathrm{Y} 3$ & $\mathrm{X} 5, \mathrm{Y} 3$ \\
\hline $\mathrm{X} 1, \mathrm{Y} 2$ & $\mathrm{X} 2, \mathrm{Y} 2$ & $\mathrm{X} 3, \mathrm{Y} 2$ & $\mathrm{X} 4, \mathrm{Y} 2$ & $\mathrm{X} 5, \mathrm{Y} 2$ \\
\hline $\mathrm{X} 1, \mathrm{Y} 1$ & $\mathrm{X} 2, \mathrm{Y} 1$ & $\mathrm{X} 3, \mathrm{Y} 1$ & $\mathrm{X} 4, \mathrm{Y} 1$ & $\mathrm{X} 5, \mathrm{Y} 1$ \\
\hline
\end{tabular}

Fig. 2. Static position measurement testing background.

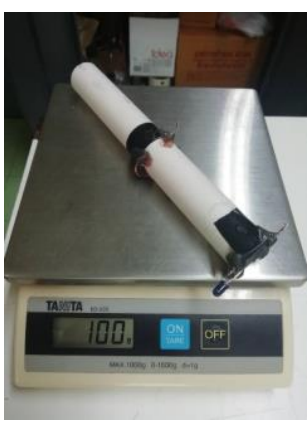

(a.)

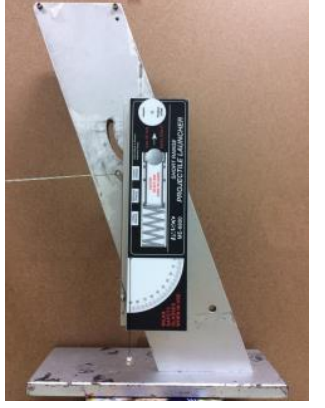

(b.)
Fig. 3. (a.) Test speciment. (b.) Projectile launcher.

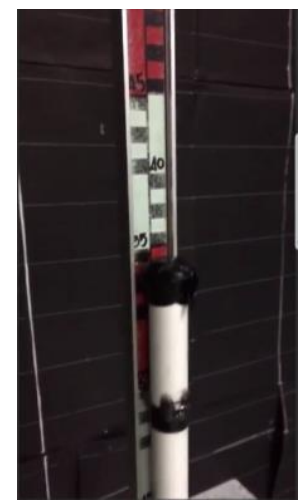

Fig. 4. Slow motion reference picture.

Real-time image processing system of infrared camera was assessed for its efficiency by using vertical camera unit beaming at a cylindrical tube which was fitted with infrared LED at the end of the tube. The tube weight was 100 grams with the dimensions of; 12.7 millimetre in diameter, and 20 centimeter in length as illustrated in Figure 3. The vertical camera unit is equipped with 3 levels of adjustable beaming power as per Figure 3.b. Subsequently, a slow-motion camera at the end of the tube was used to record VDO clips in order to determine positions of the infrared LED. The results were used as references for the height measurements which has been calibrated against level indicator that pertains the resolution of 1 centimeter as demonstrated in figure 4 .

\subsection{Measurement in accordance with the conservation of energy rules}

Measurement in accordance with the Conservation of energy rules, Counter movement jump was commenced at $\boldsymbol{t}_{\mathrm{O}}$ where the infrared LED attached belt was at its initial reference position (Ref), after that participants squat down in preparation to jump at $\boldsymbol{t}_{1}$, then took off to jump surpassing the referent level at $\boldsymbol{t}_{2}$. After reaching the highest position at $t_{3}$, participants dropped to the ground due to the gravitational force at $t_{4}$.

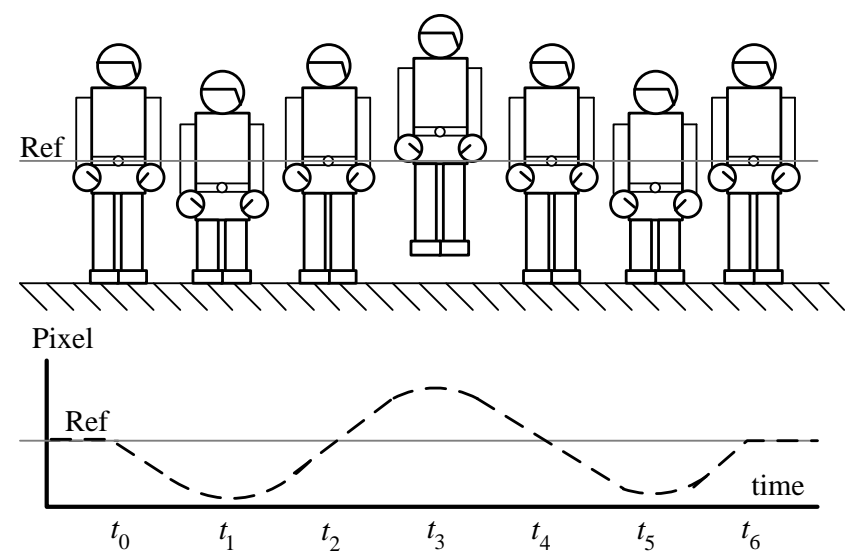

Fig. 5. Pixel coordinate-time characteristics of countermovement jump.

Energy conservation rules were used to analyze height of the jump but disregarded the initial velocity because the time used in the calculation was the entire time required to perform the jumping [18]. Conservation of energy equation is as following;

$$
\frac{1}{2} m v_{i}^{2}+m g h_{i}=\frac{1}{2} m v_{f}^{2}+m g h_{f}
$$

Prior to jumping at position Ref, there was no potential energy, and at the highest point $t_{3}$, there was no kinetic energy, therefore

$$
\frac{1}{2} m v_{i}^{2}=m g h_{f}
$$

According to the linear motion equation,

$$
v_{f}=v_{i}-g(\Delta t)
$$

$\Delta t$ Represented the entire time required in jumping which was $\boldsymbol{t}_{4}-\boldsymbol{t}_{2}$, for which timing that was recorded by the computer software was initiated at $\boldsymbol{t}_{2}$ and stopped at $\boldsymbol{t}_{4}$. This was due to the initial velocity and the final velocity of the jump were of equal value but were performed on the opposite direction. Therefore,

$$
v_{f}=-v_{i}
$$

Which could be written as;

$$
v_{i}=\frac{g(\Delta \mathrm{t})}{2}
$$


The obtained $v_{i}$ were used to calculate the height as following;

$$
h=\frac{g t^{2}}{8}
$$

\subsection{Measurement in accordance with the motion under gravity rules}

Significant factors for this measurement were; the jumping time $(t)$ and the initial velocity $(u)$. According to Figure 5, timing started from Ref position until reaching the highest point of the jump or from $t_{2}$ to $t_{3}$ which was half of the entire time required for jumping $\left(t_{4}-t_{2}\right) / 2$. Initial velocity referred to short distances that were divided by the time required for the movement within such short distance of $\left(y_{1}-y_{0}\right) / t$.

Moreover, $y_{0}$ was the initial coordinate position from Ref and $y_{1}$ referred to the coordinate position of $y_{0}$ that moved upward vertically at 100 Pixel. The initial velocity was used to calculate the height from the countermovement jump as per the motion under gravity rules as following;

$$
h=u t-\frac{1}{2} g t^{2}
$$

Half of the time was used for the calculation.

$$
h=\frac{u t}{2}-\frac{1}{2} \frac{g t^{2}}{4}
$$

Therefore, the height of countermovement jump was;

$$
h=\frac{u t}{2}-\frac{g t^{2}}{8}
$$

\subsection{Measurement by coordinate systems}

Height measurement using coordinate systems is a photographic analysis of an object's size that are generally used. The technique offers variety of applications such as measuring the flower's head of sunflower [19]. Moreover, the technique can be applied to hydrodynamic experiments [20]. Ir camera from Wii remote system can also be applied to physics experimentation [21] such as the calculation of car speed. Using the coordinate system that employs similar triangles theory to determine positions of car's headlight on both sides, the technique can determine the positions of a car that has been changed in relation to the time required for such movements [22]. In this article, similar triangles theory was used to determine the position of jumper. A belt equipped with Infrared Light-Emitting
Diodes at the buckle position with the forward Current of $100 \mathrm{~mA}$ and pertains $940 \mathrm{~nm}$ Wavelength was worn by participants in the experiment. Upon jumping from the point of reference to the highest point of jumping, displacement is considered as the vertical height of the jump that was measured for $(H)$ was related to the coordinated position captured by the photo sensor $\operatorname{array}(h)$. At the same time, the focal length of Wii remote (a) and object distance (b) remain constant. The diagram of digital image processing measuring by using the similar triangles system as shown in figure 6 .

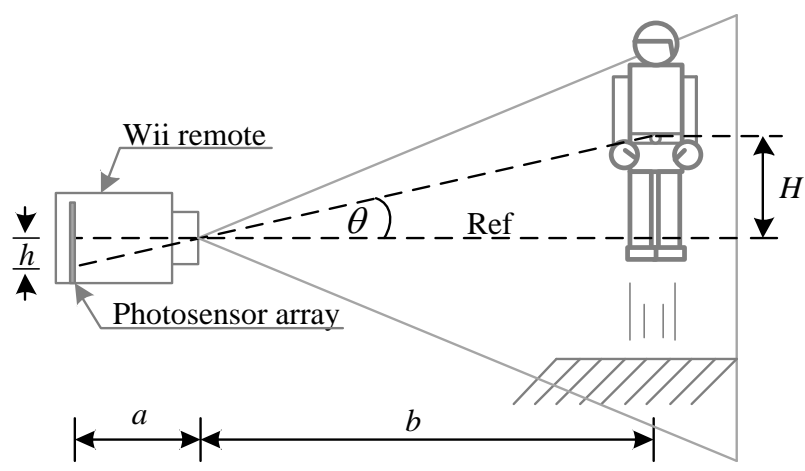

Fig. 6. Height measurement of vertical jump using similar triangles system.

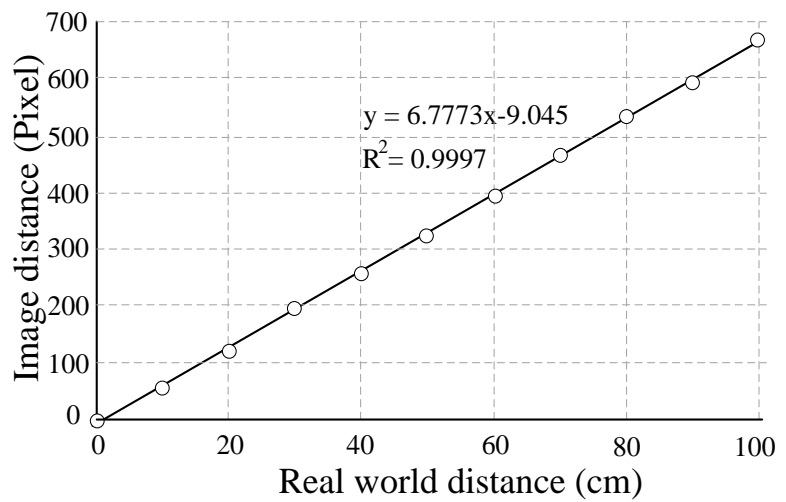

Fig. 7. Shows the relative vertical positions and the positions as recorded by the photosensor array system.

Experiments to determine the vertical change of positions were measured in centimeter while the position of photosensor array was measured in pixel. This was achieved by arranging the infrared LED at different but adjustable vertical positions. The height of such vertical arrangement was increased every 10 centimeter before the difference in height were read by the Wii remote system. The experiments were repeated 10 times.The obtained results were used to plot a graph as per Fig 7 . The horizontal axis represents an averaged value of the vertical change of the height while the vertical axis represents the positions of photosensor array that were related to the linear relationship which has $\mathrm{R}$ squared value of 0.9997 . The relationship was subsequently used to determine the vertical height that was captured by Wii remote infrared camera.

\section{Results and discussion}


System verification is a significant step that must be completed prior to measuring the height of vertical jumping. The system can be tested against an object of known value such as the positions of infrared LED which changed at different scale in both static and dynamic formats. This can be achieved by using the realtime image processing system. The system's precision was determined by measuring the height at the same spot repeatedly. For instance, the static system was measured by using Wii remote that detected the position of infrared LEDs arranged as a $5 \times 5$ matrix as per figure 2 . The test was done by switching on and off the power source that supply electricity to each infrared LED before reading the measured coordinate position of the infrared LED. The first coordination was 100,100 pixel for the subsequent increase along the $\mathrm{x}$ axis was raised to 200 pixel. Subsequently, the coordination on the y axis was raised by 100 pixel until 25 positions were completed. The tests were repeated 15 times which revealed that the highest standard deviation obtained from the measurement was 4.08 pixel as shown in Figure 8.

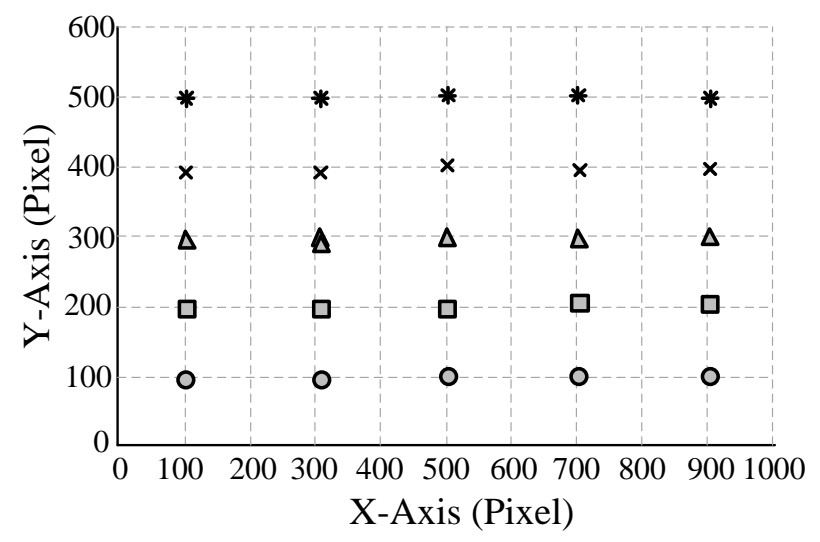

Fig. 8. Coordination recorded from the static system verification.

The assessment of real-time image processing system was recorded using veritical infared camera unit againt a cylinder tube that was attached with $5 \mathrm{~mm}$ LED which contain the forward current of $100 \mathrm{~mA}$ and $940 \mathrm{~nm}$ Wavelength at the end of the tube. The tube is $12.7 \mathrm{~mm}$ in diameter, $20 \mathrm{~cm}$ in length and was weighted down to have the total mass of 100 gram as per Figure 3.a. The vertical camera unit was adapted from PASCO's ME6800 short range projectile launcher that was adjusted to 90degree angle. The power was set at 3 level, short range, medium range and long range as illustrated in Figure 3.b. A slow motion camera was used to record the position of Infrared LED at the end of the tube against the measuring equipment that has upto 1 centimeter precision which was subseequently used as a reference for the measurement. The results of an averaged height on the measuring rod that were tested againt all 3 testing levels were shown as per table 1. Afterward, the unit was used to measure human's Counter movement vertical jump as per Figure 9 which was recorded by a slow motion camera. The results were the difference between a distance measured from the starting point (reference point) to the maximum point which was detected against the infrared LED. At this measured height, the potential energy was at its highest point where as the kinetic energy was the lowest, which was substantiated by the level indicator. The test was done with 30 male participants who were 15 basketball players and another 15 of the general public. Each of the participant repeated the jumps 15 times and the analysis was later conducted by the software-based Conservation of energy, Motion under gravity and Coordinate system as per Figure 10.

Table 1. Averaged height that was obtained from the testing

\begin{tabular}{|c|c|c|c|c|}
\hline \multirow{2}{*}{ Range } & \multicolumn{4}{|c|}{ Averaged height from the testing $(\mathrm{cm})$} \\
\cline { 2 - 5 } & $\begin{array}{c}\text { Slow } \\
\text { motion } \\
\text { camera }\end{array}$ & $\begin{array}{c}\text { Conservation } \\
\text { of } \\
\text { Energy }\end{array}$ & $\begin{array}{c}\text { Motion } \\
\text { under } \\
\text { gravity }\end{array}$ & $\begin{array}{c}\text { Coordinate } \\
\text { system }\end{array}$ \\
\hline Short & 20.60 & 19.56 & 19.56 & 19.60 \\
\hline Medium & 52.06 & 50.02 & 50.02 & 50.53 \\
\hline Long & 61.70 & 56.83 & 56.83 & 58.43 \\
\hline
\end{tabular}

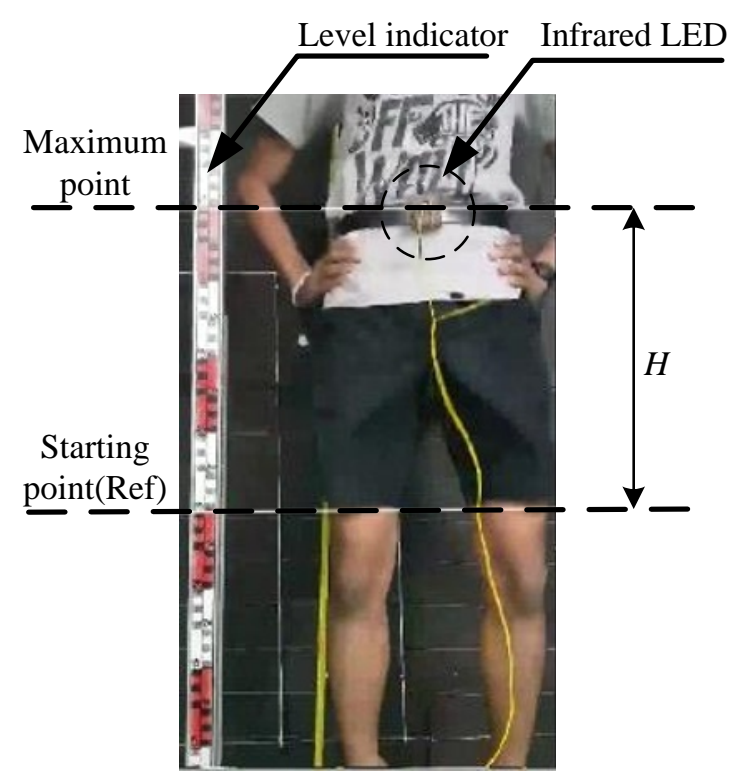

Fig. 9. Characteristics of the vertical jump as recorded from the slow motion camera.

Figure 10 illustrates two main monitors for which the top screen represents the results of the measured coordination from all the jumping and the plotted graph commenced from the moment when participants were in their position for jumping $t_{1}$, then jumped pass the set point of the software window or reference point at $t_{2}$, then passed the reference point and reached the highest position at $t_{3}$ before returning to the original position at $t_{4}$. The bottom screen indicates the time that the participants were in the air, the values that are used to calculate the height of the vertical jump. Timing was initiated when Wii remote read the vertical coordination that has a higher value at the set point of the software front panel or the referent point at $t_{2}$. Timing was stopped when Wii remote was able to read the vertical coordination which was less than the set point of the software front panel or at the reference point $\left(t_{4}\right)$. 
Subsequently, the timing was stopped and the recorded time was used to analyze the height of the vertical jump as per the principle of Conservation of energy, Motion under gravity and Coordinate system and measured heights are simultaneously displayed. Moreover, display the detail of measurement system : name of paticipate, raw data of vertical coordinate from Wii remote and hight measurand of countermovement jump from the three principles.

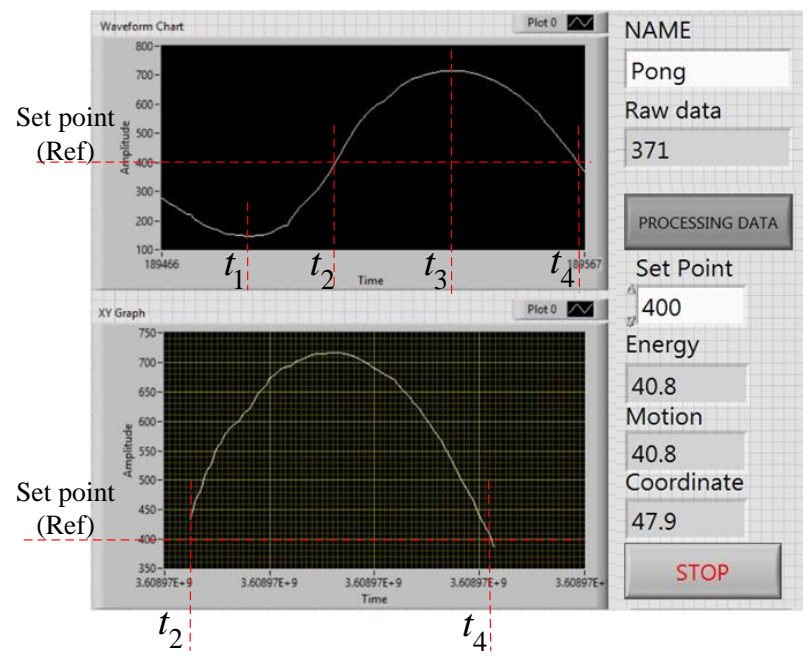

Fig. 10. The front panel of measurement software.

\section{Conclusion}

The coordination obtained by using infrared camera from Wii remote was used to analyse the vertical countermovement jump of 30 male basketball players. A software which has been developed were used for this analysis. The analysis was divided into 3 methods which were; Conservation of energy, Motion under gravity and Coordinate system. Results revealed that the Coordinate system produced the height which has the closest value to that of the reference which was determined by the motion camera. Although the deviation of this measurement was only $2.14 \mathrm{~cm}$, but the challenge of using such system is the equipment set up which requires good calibrations prior to each usage.

\section{References}

1. A.P.Webb,DJ.Taunton,D.A.Hudson,A.I.J.Forrester and S.R.Turnock, Procedia Engineering,72, Pages 709-714, (2014)

2. Sheppard JM, Dingley AA, Janssen I, Spratford W, Chapman DW, Newton RU, J Sci Med Sport, 14, Pages 85-89, (2011)

3. S.Sakka, K.Yokoi, Proceedings of the IEEE International Conference on Robotics and Automation, Pages 3752-3757, (2005)

4. S. Boukhenous, M. Attari, N. Ababou, Seventh International Symposium on Signal Processing and Its Applications, 2, Pages 13-16, (2003)

5. Walsh MS, Ford KR, Bangen KJ, Myer GD, Hewett TE, J Strength Cond Res, 20, Pages 730-734, (2006)
6. G.Annino, L.Federici, M.Gabrieli, A.Ranavolo, N.Silvaggi, V.Bonaiuto, ISEA, 147, Pages 770-775, (2016)

7. M. Rico Garcia, L.-J. Morantes Guzmán, J.-S. Botero Valencia, V. Madrid Henao, STSIVA, (2016).

8. Domire ZJ, Challis JH, J Biomech, 48, Pages 28652870 (2015)

9. Seyoung Kim, SukyungPark, Sangkyu Choi, J Biomech, 47, Pages 3162-3168, (2014)

10. Ngoh KJ, Gouwanda D, Gopalai AA, Chong YZ, J Biomech, 76, Pages 269-273, (2018)

11. Róisín Howard, Richard Conway, Andrew J Harrison, 25th IET Irish Signals \& Systems Conference and China-Ireland International Conference on Information and Communications Technologies, (2014)

12. EldarMusayev, Measurement, 39, Pages 312-319, (2006)

13. Ryan A. Pavlik, Judy M. Vance, Mechanical Engineering Conference Presentations, IOWA STATE UNIVERSITY, (2010)

14. YasharDeldjoo and Reza EbrahimiAtani, Entertainment Computing, 12, Pages 9-27, (2016)

15. S.De Amici, A.Sanna, F.Lamberti and B.Pralio, Entertainment Computing, 1, Pages 119-124, (2010)

16. Yeh CH, Hung CY, Wang YH, Hsu WT, Chang YC, Yeh JR, Lee PL, Hu K, Kang JH, Lo MT,Gait \& Posture, 43 , Pages 70-75, (2015).

17. Kevin Hejn, Jens Peter Rosenkvist, University of Copenhagen, (2008).

18. John F. Drazan, Heather Danielsen, Matthew Vercelletto, Amy Loya, James Davis, Ron Eglash, 38th Annual International Conference of the IEEE Engineering in Medicine and Biology Society, EMBC, (2016).

19. S. Sunoj, S.N. Subhashree, S. Dharani, C. Igathinathanea, J.G. Franco, R.E. Mallinger, J.R. Prasifka, D. Archer, Computers and Electronics in Agriculture, 151, Pages 403-p415, (2018).

20. Irving D. Hernandez, Jassiel V. Hernandez-Fontes, Marcelo A. Vitola, Monica C. Silva, Paulo T.T. Esperança, Ocean Engineering 157, Pages 325-p338, (2018).

21. F J Abellán, Aurelio Arenas, M J Núñez, Leandro Victoria, European Journal of Physics, 34,(2013).

22. Nils Kristian Hansen and James Robert Mitchell, IOP Publishing Ltd Physics Education, 48,(2013). 